Ann. Biol. anim. Bioch. Biophys., I969, 9 (3), 393-404.

\title{
CROISSANCE PONDERAIE DES POULETS EN FONCTION DE LA CONSOMMATION DE LA RATION DE CROISSANCE
}

\author{
J. KELLER \\ Polska Akzdemia Nauk \\ Zaktad Hodowli Doswiadczalnej \\ Zwierzat \\ Jastrzebieck/Warszzwa, \\ Pologne
}

\section{SOMMAIRE}

Grâce à une méthode d'alimentation forcée, que nous avons mise au point antérieurement, nous avons déterminé l'apport alimentaire du poussin suivant un programme fixé à l'avance. Dans cet essai, le poids sec de la ration augmente chaque jour de $\mathbf{1}, 05 \mathrm{~g}$. La composition de l'aliment est identique pour tous les animaux. Les poussins croissent alors normalement et leur composition corporelle demeure pratiquement invariable entre $4 \circ$ et I 200 grammes de poids vif.

Par ailleurs, nous avons constitué Io groupes de 5 poussins qui sont nourris comme les précédents mais, à un stade variable pour chaque groupe, le développement pondéral est arrêté en maintenant constant l'apport alimentaire. Dès lors, on peut déterminer le besoin d'entretien d'animaux de poids constant à différentes époques du développement.

Au moyen de courbes de régression, il a été établi que, contrairement aux données classiques, le besoin d'entretien est relié à la masse azotée totale par une équation linéaire.

L'importance de la masse azotée totale rend compte de l'intensité de la croissance. C'est en fonction d'elle que nous avons évalué le besoin de croissance. La part de l'aliment qui sert à couvrir les dépenses de croissance est liée au gain azoté de l'animal par une équation du second degré.

On peut théoriquement en conclure la valeur optimale du besoin de croissance.

\section{INTRODUCTION}

Dans les besoins nutritifs des animaux, on a l'habitude de distinguer une " ration d'entretien " qui correspond uniquement au processus d'existence et une "ration de production " directement transformée en produits synthétisés par l'animal, tels que lait, œufs, laine, chair, etc. Ce partage est évidemment conventionnel, quoiqu'il semble en grande partie justifié. Bien qu'il existe une étroite parenté entre les processus complexes d'entretien et de production qui se produisent dans un organisme vivant, la formation de composés chimiques multimoléculaires peut avoir lieu seulement après que soient couverts les besoins essentiels de l'animal. Elle est liée au maintien des structures cellulaires thermodynamiquement instables, aux pertes d'énergie libre occasionnées par l'activité ininterrompue des enzymes, au maintien d'une température constante de l'organisme, au travail de la circulation, des sécrétions, de la respiration et. de l'excrétion (BRODY, I945). 
On peut considérer la croissance et le développement des animaux comme un processus de production qui comprend avant tout la synthèse de nouvelles protéines et qui diffère des autres productions par le fait qu'elle s'accompagne d'une variation continuelle des besoins d'entretien de l'organisme.

Malgré le développement considérable des connaissances en nutrition au cours des dernières années, on ne connaît pas jusqu'à présent de relations quantitatives entre la taille de l'animal en croissance et ses besoins d'entretien, ni entre le gain de poids vif et la ration de croissance incluse dans les aliments consommés journellement. On admet généralement 1'existence d'une proportionnalité directe entre le gain de poids et la "ration de croissance ", mais cela n'est justifié par aucun résultat expérimental (DAvidson, I957).

L'objet de cette expérience consiste à essayer de diviser la ration journalière du poulet en croissance en "ration d'entretien " et "ration de croissance ". Par la " ration d'entretien ", nous comprenons la part de l'énergie brute de la ration qui convre seulement le processus d'existence, les besoins essentiels d'un animal. D'autre part, par la "ration de croissance ", nous comprenons la part de l'énergie brute de la ration transformée directement en produits synthétisés par le poulet en croissance, surtout en protéines.

Nous avons choisi la masse azotée corporelle des poulets comme la base commune la plus proche de la notion de " masse active du protoplasme des tissus ". Puisqu'on utilisait dans cette expérience toujours le même aliment, on pouvait exprimer la ration d'entretien et la ration de croissance en grammes de matière sèche de l'aliment.

Nous avons été amenés à rechercher l'existence de relations d'une part entre la ration d'entretien et la masse azotée du poulet, et d'autre part, entre la ration de croissance et le gain d'azote qui lui correspond.

\section{MATÉRIEL ETT MÉTHODES}

Nous avons utilisé dans cette expérience zoo poussins d'un jour de race New Hampshire. Ils étaient placés dans Io cages métalliques chauffées électriquement à une température comprise entre $26^{\circ}$ et $30^{\circ} \mathrm{C}$. La surface de chaque cage était de $0,2 \mathrm{~m}^{2}$. Au cours de l'expérience, tous les poulets ont été nourris avec un aliment complet, au moyen de la méthode d'alimentation forcée, élaborée par KielanowsKi et Keller (I962). Le mélange d'une consistance semi-liquide, introduit dans le jabot à l'aide d'une sonde, contenait en moyenne $35 \mathrm{p}$. 100 de matière sèche, et par rapport à la matière sèche, 4,23 p. I00 d'azote total, $5 \mathrm{p}$. 100 d'extrait éthéré, 3,5 p. Ioo de matières cellulosiques et 6,63 p. Ioo de cendres.

Au commencement de l'expérience, les poulets ont été répartis au hasard en deux groupes principaux de 50 individus chacun.

Le groupe $\mathrm{A}$ a été alimenté de façon que la ration journalière augmente chaque jour de la même quantité, à savoir $\mathrm{I}, 05 \mathrm{~g}$ de matière sèche. Tous les 7 jours, on sacrifiait 5 poulets de ce groupe, pris au hasard. Les animaux étaient asphyxiés à l'éther, puis autoclavés pendant environ ro heures sous une pression de $\mathbf{2 , 5}$ atmosphéres et enfin homogénéisés. On déterminait alors sur un échantillon la quantité de matière sèche, d'azote total et de cendres contenus dans le corps.

Le groupe $\mathbf{B}$ a été nourri au commencement comme le groupe'A. Mais, lorsque la ration journalière atteignait les valeurs respectives de $6,3-8,3-10,5-12,6-14,7-16,8-18,9-21,0$ - 23,1 - 25,2 g de matière sèche du mélange, on choisissait au hasard un sous-groupe de 5 poulets. Dès lors, la ration journalière de ces 5 poulets demeurait invariable. On contrôlait le gain de poids en cherchant à déterminer le moment où il devient nul. A cet effet, les animaux étaient pesés quotidiennement et on a considéré que l'arrêt de la croissance s'était produit lorsque le poids moyen clu sous-groupe n'augmentait plus pendant 5 jours consécutifs. On pouvait alors admettre que la ration journalière correspondait à la ration d'entretien. A ce moment, les poulets étaient sacrifiés et traités comme dans le groupe $\mathrm{A}$. 


\section{RÉSULTATS}

Les résultats expérimentaux sont rassemblés dans les tableaux $I, 2,3,4,5$, et 6 et sur les figures I, 2 et 3 .

TABLEAU I

Composition chimique corporelle des poulets ${ }^{(1)}$ (GROUPE A)

\begin{tabular}{c|c|c|c}
\hline \hline $\begin{array}{c}\text { Poids vif } \\
(\mathrm{g})\end{array}$ & $\begin{array}{c}\text { Matière sèche } \\
(\mathrm{g})\end{array}$ & $\begin{array}{c}\text { Azote total } \\
(\mathrm{g})\end{array}$ & $\begin{array}{c}\text { Cendres totales } \\
(\mathrm{g})\end{array}$ \\
39,4 & $\mathbf{1 0 , 1 1}$ & $\mathbf{1 , 1 4 3}$ & $\mathbf{0 , 7 6 6}$ \\
$\mathbf{5 4 , 4}$ & $\mathbf{1 2 , 5 4}$ & 1,391 & 1,346 \\
102,8 & 29,37 & 2,967 & 2,775 \\
188,8 & 54,79 & 4,975 & $\mathbf{5 , 4 4 0}$ \\
291,6 & 90,21 & 8,160 & 9,816 \\
418,0 & 130,32 & 11,167 & 14,443 \\
580,2 & 187,87 & 16,619 & 19,668 \\
$\mathbf{6 9 5 , 8}$ & 229,87 & $\mathbf{2 1 , 9 6 9}$ & 27,694 \\
903,3 & 295,66 & 28,188 & $\mathbf{3 4 , 9 5 2}$ \\
1031,6 & 355,01 & 32,204 & 39,166 \\
\hline
\end{tabular}

(1) Moyennes des sous-groupes (pour 5 poulets).

\begin{tabular}{|c|c|c|c|}
\hline \multicolumn{4}{|c|}{$\begin{array}{c}\text { TABLEAU } 2 \\
\text { Composition chimique corporelle des poulets }(1)\end{array}$} \\
\hline $\begin{array}{l}\text { Poids vif } \\
(\mathrm{g})\end{array}$ & $\begin{array}{l}\text { Matière sèche } \\
(\mathrm{g})\end{array}$ & $\begin{array}{l}\text { Azote total } \\
\quad(\mathrm{g})\end{array}$ & $\begin{array}{l}\text { Cendres totales } \\
(\mathrm{g})\end{array}$ \\
\hline 101,8 & 26,73 & 3,30 & 3,48 \\
\hline 109,2 & 28,18 & 3,47 & 4,03 \\
\hline 179,0 & 51,02 & 6,19 & 6,58 \\
\hline 193,4 & 53,90 & 6,57 & $7, \mathbf{4 3}$ \\
\hline 395,0 & 113,42 & 15,43 & 16,67 \\
\hline 450,0 & 130,38 & 16,69 & 20,78 \\
\hline 546,2 & 153,17 & 21,10 & 23,74 \\
\hline 585,8 & 160,10 & 20,44 & 26,03 \\
\hline 651,8 & 186,11 & 25,80 & 29,40 \\
\hline 757,6 & 219,00 & 27,84 & 34,03 \\
\hline
\end{tabular}

(1) Moyennes des sous-groupes (pour 5 poulets).

On a soumis les données à l'analyse statistique pour étudier la liaison entre la ration journalière et la taille maximale du poulet qu'elle permet. La taille du poulet a été exprimée par la masse azotée qui devait être proportionnelle à la masse active du protoplasme des tissus.

Comme les rations successives d'aliments utilisées dans le groupe $B$ diffèrent de la même valeur fixe $(2, \mathrm{I} \mathrm{g})$, on a pu calculer la régression en se servant de la méthode des polynômes orthogonaux. La régression est constituée par une droite dont l'équation s'écrit :

$$
y=-7,73+\mathrm{I}, 424 z
$$

où $y=$ masse azotée corporelle du poulet en $\mathrm{g}$,

$z=$ ration d'entretien en $g$ de matière sèche. 
TABLEAU 3

Analyse de variance avec des effets curvilinéaires (1) (GROUPE B)

\begin{tabular}{|c|c|c|c|c|}
\hline Variabilité & Degrés de liberté & Somme des carrés & Carré moyen & F trouvé \\
\hline 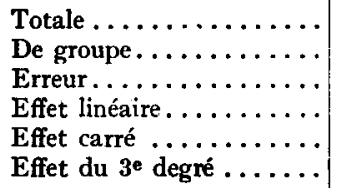 & $\begin{array}{r}49 \\
9 \\
40 \\
1 \\
1 \\
1\end{array}$ & $\begin{array}{r}4065,92 \\
3844,00 \\
221,98 \\
3686,44 \\
1,96 \\
6,96\end{array}$ & $\begin{array}{l}5,55 \\
1,96 \\
6,96\end{array}$ & $\begin{array}{l}0,35 \\
1,25\end{array}$ \\
\hline
\end{tabular}

F théoriquues: $\mathrm{F}_{0,05}=4,08 \quad \mathrm{~F}_{0,01}=7,31$.

(1) Concerne la régression : $y=-7,73+1,424 z$.

On a également calculé statistiquement la régression inverse, nécessaire pour les considérations qui suivent. Le tableau 4 rassemble les calculs et la figure I représente les données.

L'équation obtenue s'écrit:

$$
z=6,394+0,6369 y
$$

TABLEAU 4

L'évaluation de la régression : $z=6,394+0,63^{6} 9$ y (GROUPE B)

\begin{tabular}{|c|c|c|}
\hline $\begin{aligned} \mathrm{E}_{y} & =\quad \begin{array}{c}734,5 \\
\mathrm{E}_{y}^{2}\end{array}=14855,73\end{aligned}$ & $\mathrm{E}_{y z}=14157,99$ & 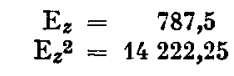 \\
\hline$\frac{1}{\mathrm{n}} \mathrm{E}^{2} y=10789,81$ & $\frac{1}{n} E_{y} E_{z}=11568,38$ & $\frac{1}{\mathrm{n}} \mathrm{E}^{2}{ }_{z}=12403,13$ \\
\hline 4065,92 & $S_{y z}=2589,61$ & 1819,12 \\
\hline
\end{tabular}

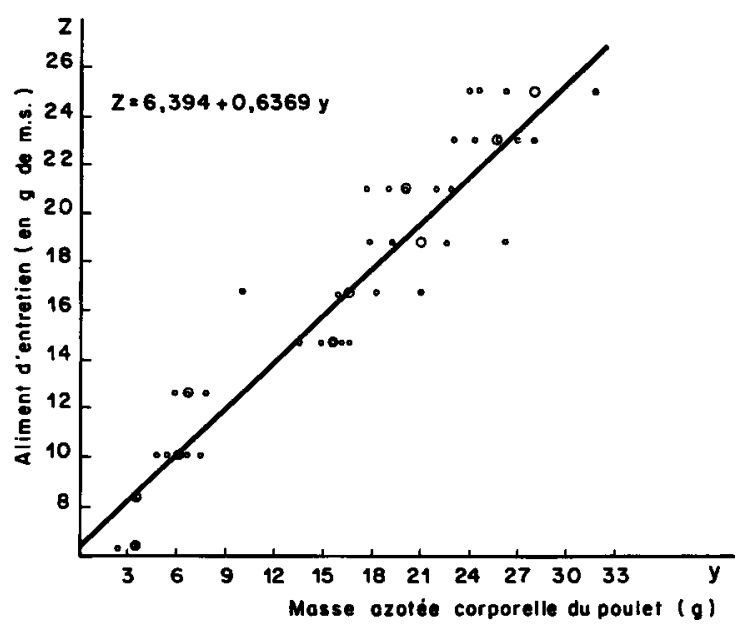

FIG. I. 
En analysant statistiquement de la même manière les données obtenues pour le groupe $A$, on a établi l'existence d'une régression entre l'âge et la masse azotée des poulets (tabl. 5 et fig. 2). L'équation s'écrit, avec une exactitude suffisante :

$$
y=0,565-0,0298 t+0,007 \text { I3 } t^{2}
$$

où $y=$ masse azotée corporelle du poulet en $\mathrm{g}$,

$t=$ âge du poulet en jours.

\section{TABLEAU 5}

Analyse de variance avec des effets curvilinéaires (') (GROUPE A)

\begin{tabular}{|c|c|c|c|c|}
\hline Variabilité & Degrẽs de liberté & Somme des carrés & Carré moyen & F trouvé \\
\hline 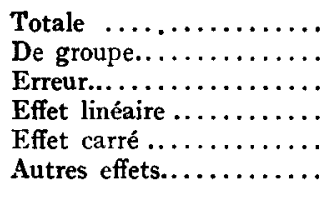 & $\begin{array}{r}49 \\
9 \\
40 \\
1 \\
1 \\
7\end{array}$ & $\begin{array}{r}5897,03 \\
5801,95 \\
85,08 \\
5457,09 \\
322,70 \\
22,14\end{array}$ & 2,1 & 1,5 \\
\hline
\end{tabular}

Fthéoriques : $F_{0,05}=4,08 \quad F_{0,01}=7,31$.

(1) Concerne la régression : $y=0,565-0,0298 t+0,00713 t^{2}$.

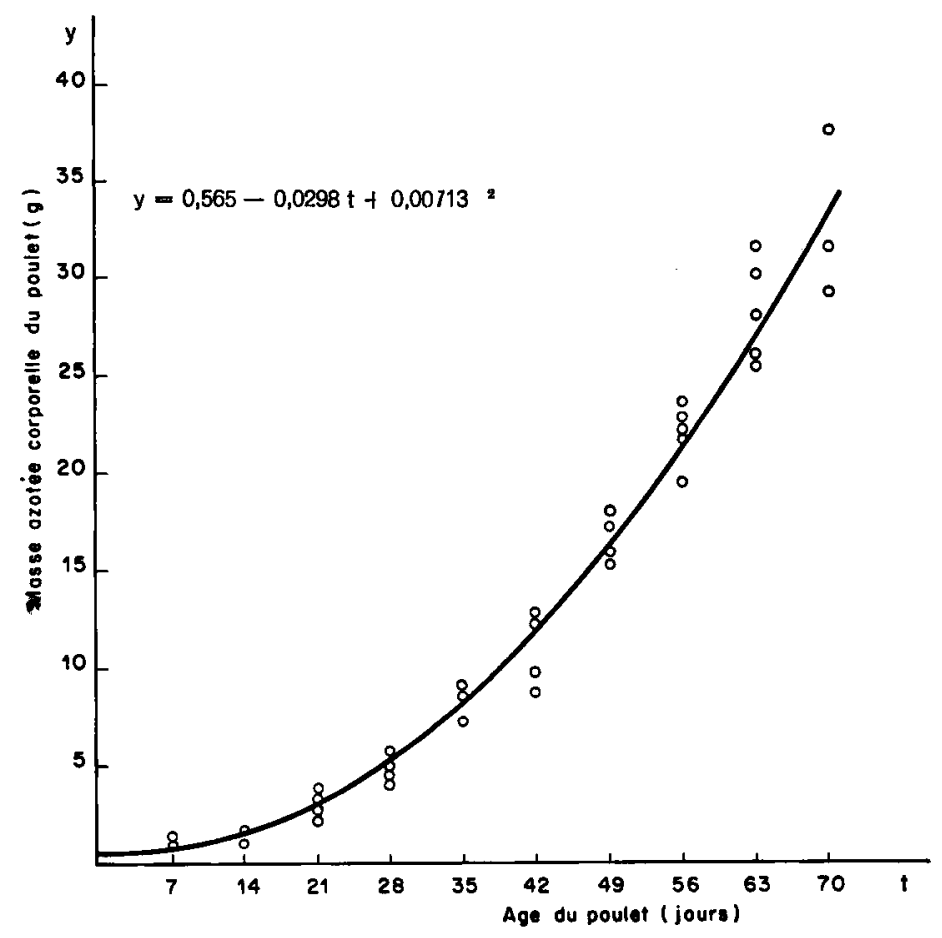

FIG. 2. 
De ces deux équations fondamentales de régression obtenues dans cette expérience, on peut tirer la valeur de $z$ en fonction de $t$ :

$$
z=6,754-0,019 t+0,00454 t^{2}
$$

La ration journalière pour le groupe $A$ constitue une fonction linéaire du temps établie $a$ priori et se traduisant par l'équation :

$$
w=\mathrm{I}, 05 t
$$

où $w=$ ration journalière en $\mathrm{g}$ de matière sèche,

$t=$ âge du poulet en jours.

On peut alors calculer la fonction $q$, différence entre les équations (4) et (3) :

$$
q=w-z=-6,754+\mathrm{I}, 069 t-0,00454 t^{2}
$$

où $q=$ ration de croissance contenue dans les aliments distribués chaque jour en $\mathbf{g}$ de matière sèche.

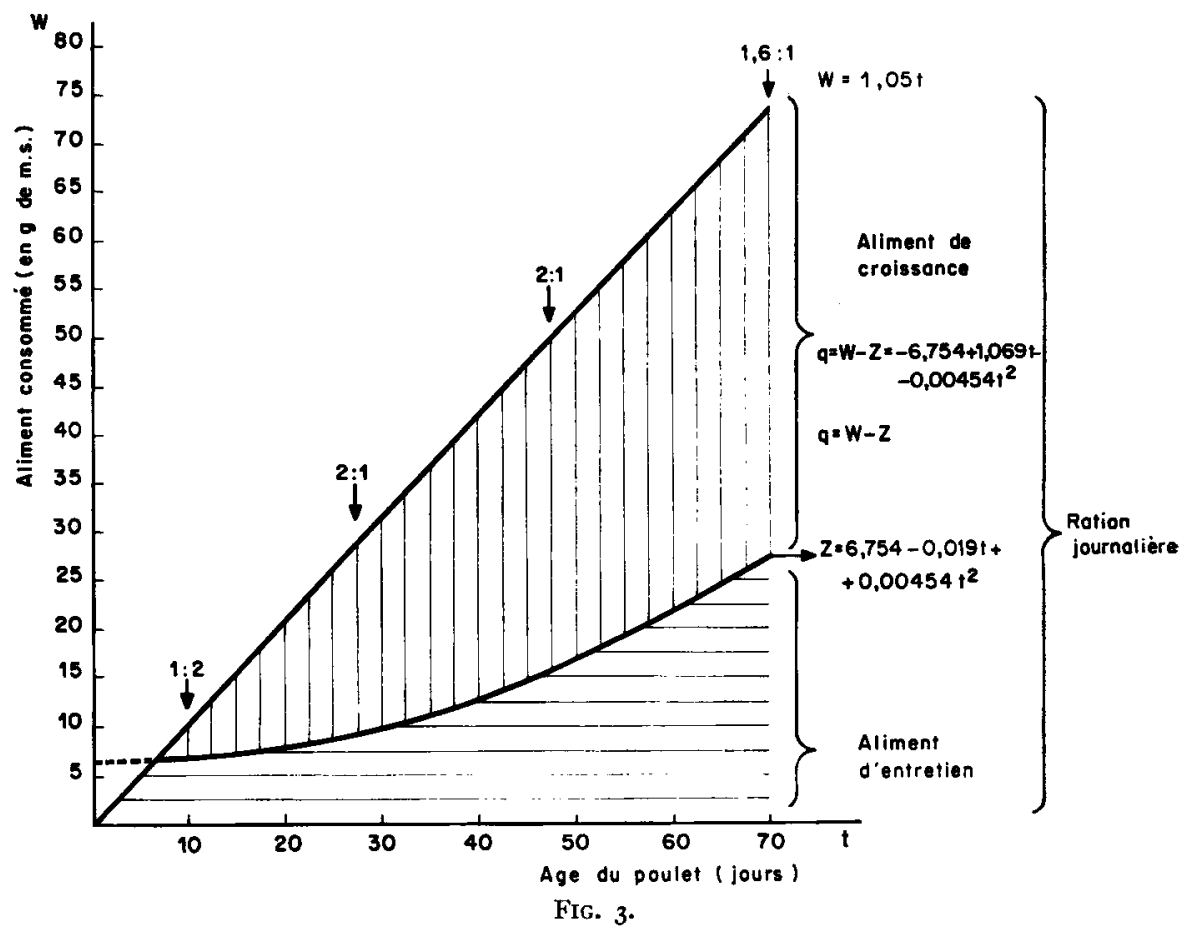

La dérivée de la fonction (2) représente la rétention azotée journalière du poulet; son expression est :

$$
y^{\prime}=r=-0,0298+0,0{ }_{4} 26 t
$$

où $r=$ rétention azotée journalière corporelle.

La fonction inverse (transformation algébrique) exprime la relation entre l'âge du poulet en jours et la valeur journalière de la rétention azotée corporelle en grammes. Elle s'exprime par l'équation :

$$
t=2,09+69,93 r
$$


En reportant cette valeur de $t$ dans l'équation (5), on exprime la relation qui existe entre la ration de croissance journalière en $\mathrm{g}$ de matière sèche et la rétention journalière d'azote du poulet :

$$
q=-4,54+73,43 r-22,202 r^{2}
$$

\section{DISCUSSION}

\section{Influence de l'arrêt de la croissance des poulets sur le développement de quelques-uns de leurs organes internes}

Les résultats et déductions énoncés ici supposent que la méthode utilisée dans cette expérience pour arrêter la croissance des poulets n'exerce aucune influence sur le jéveloppement relatif des différents organes et par suite, ne change pas les besoins d'entretien. Il est bien sûr impossible de donner une réponse définitive. Mais il existe un certain nombre de faits qui répondent à cette question.

A partir des premiers travaux de WATERS (cités par HAMmond, I955), en passant par les expériences classiques de MCMkEKan (I940) et jusqu'aux recherches de WILSON (I952 et I954), de nombreux auteurs se sont efforcés d'étudier l'influence du niveau d'alimentation sur la croissance pondérale et le développement de certains compartiments corporels des animaux. Ils admettent que le changement de niveau de nutrition durant la croissance exerce l'influence la plus grande sur l'organe ou le tissu qui, dans la période considérée, est caractérisé par la capacité maximale de croissance.

Wirson, déjà cité, réalise ses expériences sur poulets, d'après le schéma de McMEEKAN. Il a d'abord constaté une influence du niveau d'alimentation sur la constitution anatomique des poulets en les comparant au même poids vif. Mais plus tard, il s'est rétracté.

Les expériences de KELLER (I963) et KELLER et al. (I966 a), montrent chez le poulet que le niveau d'alimentation influence la taille du foie et dans une faible mesure celle du tube digestif. Le foie représente environ 3 p. roo de la masse corporelle chez les animaux à l'entretien contre 3,7 p. Ioo pour ceux du groupe nourris à un niveau élevé. Cependant la croissance des principaux groupes de muscles du squelette n'est pas affectée. La proportion des muscles pectoraux et de la cuisse par rapport au poids du poulet reste généralement constante et ne dépend pas de la race au moins jusqu'à $500 \mathrm{~g}$ de poids vif, soit $9,6 \mathrm{p}$. Ioo pour les premiers et I4,3 p. Ioo pour les seconds. En déterminant le pourcentage d'azote dans les muscles, le foie et le tube digestif des poulets nourris à des niveaux différents, on n'a constaté aucune influence marquée du niveau d'alimentation quoique les analyses portent sur 60 individus d'âges différents et de poids compris entre 50 et I $200 \mathrm{~g}$.

D'un point de vue pratique, on peut donc considérer que notre mode d'alimentation ne modifie pas les proportions des différents organes des poulets nourris avec des quantités très différentes d'un même régime. Il est possible que dans d'autres conditions expérimentales il en soit autrement.

Nous avons antérieurement démontré que notre mode d'alimentation maintient la composition corporelle égale à elle-même quel que soit le poids de l'animal en croissance dans la zone étudiée (40-I $200 \mathrm{~g}$ ). 


\begin{tabular}{|c|c|c|}
\hline & \multicolumn{2}{|c|}{ Alimentation } \\
\hline & intensive & extensive \\
\hline 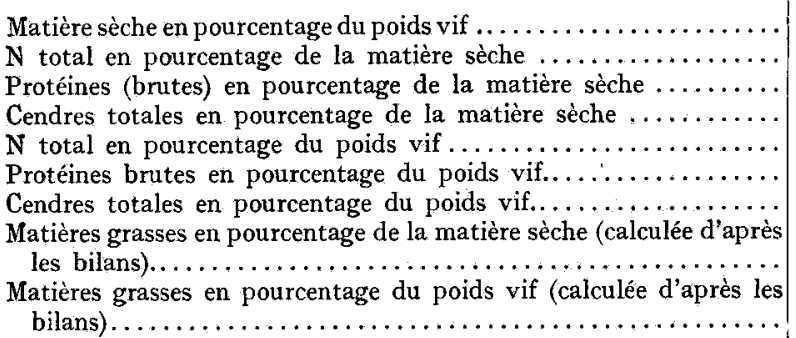 & $\begin{array}{r}32,42 \\
9,23 \\
57,69 \\
11,18 \\
2,99 \\
18,69 \\
3,62 \\
\\
30,00 \\
10,00\end{array}$ & $\begin{array}{r}28,26 \\
13,09 \\
81,81 \\
15,34 \\
3,70 \\
23,13 \\
4,34 \\
3,00 \\
1,00\end{array}$ \\
\hline
\end{tabular}

D'autre part, il est bien évident que le niveau d'alimentation influence la composition chimique des corps des poulets (tabl. 6). Nos propres travaux (KELLER et coll., I967 a) le confirment nettement. Les poulets nourris intensivement sont plus gros que les témoins nourris extensivement. On pourrait donc supposer que les dépenses "énergétiques liées au maintien des protéines et celles de la graisse sont différentes. Mais ce n'est pas vrai. Schrzmann (1958) a calculé que les dépenses énergétiques d'un animal, liées au maintien de sa graisse corporelle sont les mêmes que celles liées au maintien de tous ses tissus. Donc cela confirme notre principe que la masse azotée du corps est proportionnelle à la masse active du protoplasme des tissus.

\section{Besoins d'entretien des poulets pendant la croissance}

La détermination du besoin d'entretien chez les animaux adultes constitue un problème relativement simple, déjà résolı par KELLNER à la fin du XIX ${ }^{\mathrm{e}}$ siècle et au début du $\mathrm{xx}^{\mathrm{e}}$. En revanche, chez les animaux en croissance, il n'existe pratiquement aucune expérience de détermination de ce besoin, abstraction faite des mesures de métabolisme de base qui s'effectuent chez l'animal au repos, à jeun et à la neutralité thermique, ce qui correspond pour le poulet à un retrait des aliments pendant 24 heures et à une température comprise entre 15 et $25^{\circ} \mathrm{C}$. Dans les conditions normales d'élevage, les animaux ne se trouvent ni au jeûne, ni au repos complet, bien que la liberté de mouvement soit quelquefois réduite. Aussi leurs dépenses d'énergie, en envisageant seulement le maintien de la vie, diffèrent notablement de celles qui sont trouvées dans les conditions particulières de la détermination du métabolisme de base. Il en résulte que ce dernier ne peut correspondre au besoin d'entretien de l'animal.

C'est en partant de ces considérations que nous avons élaboré notre méthode d'estimation du besoin d'entretien chez le poulet en croissance. Elle consiste à déterminer le poids maximal de l'oiseau pour une quantité journalière d'aliment déterminée a priori en estimant que, lorsque ce poids est atteint, la ration quotidienne ne contient aucun surplus permettant la synthèse de nouveaux constituants corporels 
supplémentaires (KE,LER et al., Ig66 b). Ce poids maximal correspond à l'établissement d'un bilan azoté nul, comme nous l'a montré une autre expérience. Le poids maximal est facilement déterminé car la courbe de croissance des animaux est très régulière en raison du mode de distribution des aliments. En outre, dans une autre étude (KELLER et $a l, \mathrm{I}_{967} b$ ) nous avons distribué la même quantité d'aliment chaque jour à des poulets dont les poids étaient dans le rapport du simple au triple. Tous ont atteint le même poids maximal, mais au bout d'un temps plus ou moins long.

L'étude des résultats obtenus montre que le besoin d'entretien augmente avec le poids du poulet. Il est directement proportionnel à la masse azotée corporelle pour des animaux en croissance, élevés en liberté dans des cages de $40 \mathrm{sur} 50 \mathrm{~cm}$, à la température de $26^{\circ}$ à $30^{\circ} \mathrm{C}$ et recevant 3 repas par jour. Mais nos études antérieures, citées plus haut, nous montrent que l'abaissement de la température au-dessous de $26^{\circ} \mathrm{C}$ entraîne une augmentation des dépenses énergétiques des poulets, influence le poids maximal final et par suite la régression obtenue.

Ainsi, en respectant un certain nombre de conditions relatives à l'élevage et au mode d'alimentation, nous estimons possible la mesure du besoin d'entretien du poulet selon une méthode relativement simple.

Cette méthode est évidemment conventionnelle. Si l'arrêt de la croissance des poulets durait plus longtemps, la composition chimique corporelle varierait. Dans notre expérience on sacrifiait les animaux aussitôt que possible, toujours de la même façon. On peut donc croire que les conditions standardisées ont permis d'éviter le phénomène constaté par KAUNITz et al. (I956) chez les rats selon lequel 1'arrêt prolongé de la croissance provoque des modifications du développement des organes internes.

\section{Gain corporel d'azote en fonction de la ration de croissance journalière}

I a plupart des unités d'évaluation de la valeur énergétique des aliments repose sur l'existence d'une proportionnalité directe entre le gain de poids ou de substances énergétiques et l'énergie potentielle contenue dans la ration lorsque les besoins d'entretien sont couverts. Cela s'applique aussi bien à l'Unité Amidon de KELLLNER, qu'à l'Unité Fourragère scandinave de NILs-Hansson, où française de L,EROY, ou à 1'Unité Avoine communément employée en Pologne.

Pour les volailles, ce principe a été utilisé par FRAPS (d'après DAvidson, I957) pour calculer l'énergie productive des aliments entrant dans le régime des oiseaux; cette unité correspond à la quantité d'énergie fixée par l'organisme sous forme de protides et de lipides correspondant à un gramme d'aliment consommé au-delà de la couverture du besoin d'entretien. La méthode algébrique de détermination suppose en outre la proportionnalité directe de la ration de croissance par rapport au gain de poids. Ce principe ne repose sur aucune base expérimentale.

La relation (7) établie à partir de nos résultats expérimentaux nous indique que la ration journalière de croissance diminue relativement, par rapport à $\mathrm{I} g$ de $\mathrm{N}$ retenu, quand le gain corporel azoté augmente (fig. 4). La proportionnalité directe entre ces deux grandeurs n'existe pas. Dès lors, on comprend pourquoi Davioson et al. (I957) constatent que l'énergie productive de FRAPS n'est pas toujours additive. Cela dépend de la vitesse de croissance des animaux en fonction du niveau alimentaire.

Annales de Biologie animale. - I969. 
Il nous est également possible de calculer la croissance pondérale qui permet l'utilisation optimale de la ration de croissance. En dérivant l'équation (7), on obtient la ration journalière qui correspond à I $\mathrm{g}$ d'augmentation de la masse azotée corporelle durant l'expérience :

$$
q^{\prime}=73,43-44,404^{r}
$$

L'utilisation maximale correspond à l'annulation de cette dérivée. Nous obtenons la valeur de $\mathrm{I}, 654 \mathrm{~g}$ pour la rétention corporelle d'azote, soit une augmentation quotidienne de poids de $50 \mathrm{~g}$ environ, dans les conditions de notre expérience.

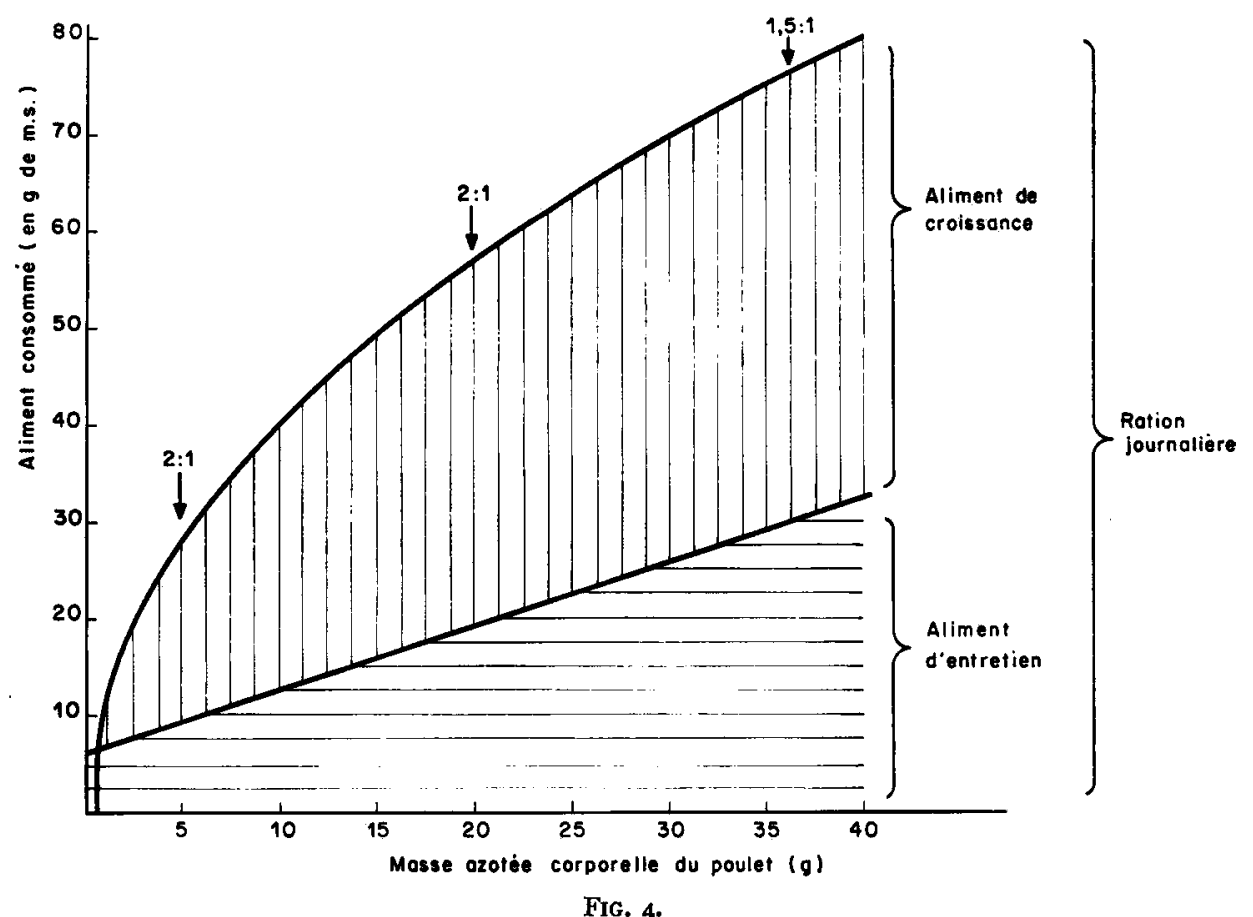

\section{CONCLUSION}

L'ensemble de nos travaux constitue une des premières tentatives de mesure des besoins d'entretien et de croissance du poulet à partir de résultats expérimentaux. Les équations que nous avons pu formuler ont montré que :

- le besoin d'entretien est une fonction linéaire de la masse azotée corporelle;

- la relation entre la ration journalière de croissance et le gain quotidien de protides corporels est curvilinéaire.

Ces conclusions ont pu être tirées grâce à notre méthode d'alimentation, car l'augmentation régulière de l'ingéré distribué en trois repas forcés quotidiens ne provoque aucune modification de la composition corporelle au cours de la croissance.

Comme les autres conditions expérimentales sont proches de la pratique, nous estimons possible de déterminer la croissance qui correspond à la meilleure utilisation 
des aliments. Pour la souche que nous avons utilisée - New Hampshire - nous avons trouvé que ce gain de poids journalier devait se situer aux environs de $50 \mathrm{~g}$.

Reçu pour publication en mars r969.

\section{SUMMARY}

\section{WEIGHT INCREASE IN CHICKENS IN RELATION TO INTAKE OF THE GROWING RATION}

By the method of forced feeding previously described (KIELANOwSKI and KELLER, I962) the supply of feed to chickens was fixed according to a predetermined programme. In this trial the dry matter of the ration was increased by $1.05 \mathrm{~g}$ per day. Composition of the feed was the same for all the birds. The chickens grew normally and their body composition remained practically the same between $4^{\circ}$ and $\mathrm{r}, 200 \mathrm{~g}$ liveweight.

Also, to groups of 5 chickens were fed as the previous ones but at a different stage for each group weight gain was stopped by keeping the food supply constant. From there it was possible to estimate the maintenance requirement of birds of constant weight at different stages of development.

By means of regression lines it was shown that, contrary to the classical data, the maintenance requirement $(z)$ is not proportional to liveweight of the chicken but is related to total nitrogen of the body $(y)$ by a linear equation :

$$
z=6.394+0.6369 y
$$

The amount of total nitrogen takes into account the intensity of growth. It is in relation to this that the maintenance requirement has been evaluated. The portion of the feed which covers the cost of growth $((q)$ is related to the gain of nitrogen by the bird $(r)$ by a second degree equation :

$$
q=4.54+73.43 r-22.202 r^{2}
$$

Optimum value for the maintenance requirement can be established theoretically.

\section{RÉFÉRENCES BIBLIOGRAPHIQUES}

BroDY S., 1945. Bioenergetic and Growth.

Davidson J. M., Donald J., Whlliams R. B., 1957. The utilization of dietary energy of poultry. Sci. Food Agric., 8, 3 .

Hammond J., I955. Progress in the physiology of farm animals.

Kaunitz H., Slanetz C. A., Johnson R. E., Guillmain J., I956. Influence of diet composition on caloric requirement, water intake and organ weights of ratio during restricted food intake. J. Nutr., 60, $221-228$.

Keller J., 1963. Croissance des poulets en fonction de la consommation d'aliments. Diss.

Keller J., Keller A., I966. Influence of inter-breed differences upon the growth rate and body proportions in chickens. Bull. Inst. Elev. Exp. Anim. dom. Acad. Pol. Sci., nº 9.

KELLER J, KELLER A., r 966 . Influence of inter-breed difference upon the maintenance requirements of growing chickens. Bull. Inst. Elev. Anim. dom. Acad. Pol. Sci., no 9.

Keller J., RudzkA J., I967. A contribution to the method of establishing the maintenance feed in the nutrition of chickens. Bull. Inst. Elev. Exp. Anim. dom. (sous presse).

KELler J., RUdzkA J., Duchinska J,, GNIADZIK K., I967. Influence of feeding level upon the chemical composition in chicken's carcasses. Bull. Inst. Élev. Anim. dom. Acad. Pol. Sci. (sous presse).

Kielanowski J., Keller J., rg62. Eine Methode quantitativer Einzelfütterung von Küken. Arch. Tierernährung, 12, 5 .

McMeekan C. P., 1940. Growth and developopement in the pig with special reference to carcass quality character. J. Agric. Sci., 30, 4 .

SchikmanN R., 1958. Kritische Betrachtungen über die Entwicklung der Stärkewertlehre Oskar Kellners. Disch. Akad. Landwirt. Wiss. Ab., no $3 \mathbf{I}$.

Wilson P. N., I952. Growth analysis of the domestic fowl. I. Effect of plane of nutrition and sex on liveweight and external measurements. J. Agric. Sci., 42.

Wilson P. N., I954. Growth analysis of the domestic fowl. II. Effect of plane of nutrition on carcass composition. J.Agric. Sci., 44. 